Article

\title{
Ultra-Wideband Low-Cost High-Efficiency Cavity-Backed Compound Spiral Antenna
}

\author{
Charl Baard ${ }^{\dagger}$, Yulang Liu ${ }^{\dagger}$ and Natalia Nikolova ${ }^{*}+$ \\ Department of Electrical and Computer Engineering, McMaster University, Hamilton, ON L8S 4K1, USA; \\ baardc@mcmaster.ca (C.B.); liuy461@mcmaster.ca (Y.L.) \\ * Correspondence: nikolova@ieee.org \\ + These authors contributed equally to this work.
}

Received: 20 July 2020; Accepted: 26 August 2020; Published: 28 August 2020

\begin{abstract}
A low-cost high-efficiency ultra-wideband (UWB) cavity-backed spiral antenna is proposed. It employs an equiangular spiral enclosed by an Archimedean spiral and it is fed through a tapered microstrip balun. A center-raised cylindrical absorber-free cavity backs the spiral to minimize the backward radiation without decreasing the efficiency. The cavity is designed to ensure an impedance bandwidth exceeding 16:1 ratio (from $350 \mathrm{MHz}$ to $5.5 \mathrm{GHz}$ ). Simulated and measured results are presented and compared, demonstrating competitive performance in terms of impedance bandwidth and efficiency. Time-domain measurements indicate fidelity of 0.62 at boresight.
\end{abstract}

Keywords: ultra-wideband antennas; spiral antennas; cavity backed spiral antennas

\section{Introduction}

Imaging and detection radars [1-3] often employ narrow pulses with frequency bandwidths of 10:1 or more. They need antennas with impedance and radiation characteristics that must meet challenging ultra-wideband (UWB) design specifications.

Among the various types of UWB antennas, the spiral is a prime choice when circular polarization $(\mathrm{CP})$ is needed [4-12]. In imaging and detection, wave diversity in terms of right-hand (RH) and left-hand (LH) CP is beneficial because it differentiates the back-scatter of smooth large surfaces from that of wires, edges and electrically small objects $[6,13]$. Also, the spiral antennas can be fabricated on printed circuit boards (PCBs) making them mechanically robust and relatively cheap.

On the other hand, the spiral antennas have some disadvantages. They require a balanced feed to a relatively high input impedance, e.g., about $180 \Omega$ to $210 \Omega$ for a free-standing two-arm printed spiral [9], or, about $120 \Omega$ for a two-arm slot spiral [14]. Thus, a UWB balun is needed to match the typical $50-\Omega$ feed to the spiral's input. High-permittivity thick substrates can reduce the spiral's input impedance but at the cost of narrower impedance and axial-ratio (AR) bandwidths $[15,16]$. Also, designing a spiral antenna for unidirectional radiation comes at a price. Metallic reflectors or cavities are usually used [7-10,14,17-30]. The cavity adversely affects the impedance and the AR bandwidths as well as the gain-pattern symmetry $[18-20,27]$. This is often mitigated by the insertion of absorbing materials or electromagnetic band-gap (EBG) structures [26]. The EBG solution is not feasible when the impedance bandwidth must exceed 10:1 ratios. On the other hand, the absorbers decrease the radiation efficiency, which is particularly unwanted when low-cost laminates (e.g., FR-4) are used since their loss is already high. The laminate cost is important when the antenna is large (frequencies of operation are low), which is the case considered here.

The proposed design employs a compound spiral [28-34], which consists of an equiangular spiral enclosed by an Archimedean one (see Figure 1). Compound spirals are a compromise between the better conductor radiation efficiency of the equiangular spiral and the smaller size of the Archimedean 
one. However, achieving unidirectional radiation with a compound spiral is challenging $[28,35,36]$. In [28], simulations demonstrate the use of a complex cavity, which employs a ladder-shaped bottom along with six cylindrical metallic fins whose edges are coated with absorptive films. In [35,36], absorber-free cavities are used but the spiral arms are terminated with resistors. Here, we show that an absorber-free cylindrical cavity of simple center-raised geometry can provide unidirectional patterns and very wide (16:1) impedance bandwidth from $350 \mathrm{MHz}$ to $5.5 \mathrm{GHz}$ while avoiding additional losses. Efficiency between $58 \%$ and $78 \%$ is achieved in the whole frequency band despite the low-cost FR-4 substrate $(\tan \delta \approx 0.02)$ whereas the typical efficiency of spiral antennas with absorptive backing (even if fabricated on high-cost substrates with $\tan \delta \approx 9 \cdot 10^{-4}$ ) is $40 \%$ to $45 \%$ [20,37]. The minimum AR (within $45^{\circ}$ of boresight) is better than $5 \mathrm{~dB}$ from $500 \mathrm{MHz}$ to $5 \mathrm{GHz}$. On the other hand, the absorber-free cavity affects adversely the uniformity of the gain and AR patterns, especially at higher frequencies.

\section{Antenna Structure and Design Approach}

The proposed antenna consists of three main parts: compound spiral, cavity and balun (see Figure 1). The design requirements in the bandwidth from $500 \mathrm{MHz}$ to $5 \mathrm{GHz}$ are: (i) return loss of $10 \mathrm{~dB}$ or better, (ii) front-to-back ratio of $6 \mathrm{~dB}$ or more, and (iii) minimum AR within $45^{\circ}$ of boresight less than $6 \mathrm{~dB}$. The antenna must be low-cost and easy to fabricate. It is designed using HFSS [38]. CST [39] is used for verification. The design shown here is for right-hand polarization. We have also implemented the analogous left-hand polarized antenna, the performance of which is very similar to the results reported here in terms of impedance bandwidth, gain and axial ratio.

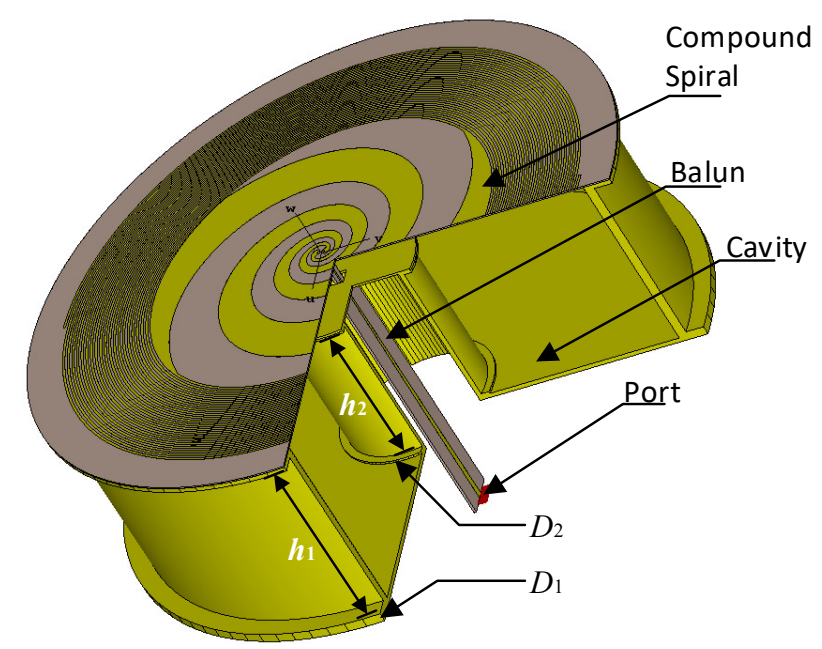

Figure 1. Overall view of the cavity-backed compound-spiral antenna structure.

\subsection{Compound Spiral}

The compound spiral (Figure 2) is printed on a $1 \mathrm{~mm}$ thick FR-4 PCB with a diameter of $367 \mathrm{~mm}$. The expressions defining the edges $r_{e 1}$ and $r_{e 2}$ of one of the equiangular-spiral arms are

$$
\begin{aligned}
& r_{e 1}=r_{0} \exp \left(\alpha_{e} \theta_{e 1}\right), \\
& r_{e 2}=r_{0} \exp \left(\alpha_{e} \theta_{e 2}\right)
\end{aligned}
$$

where $r_{0}=5 \mathrm{~mm}, \alpha_{e}=0.25,0 \leq \theta_{e 1} \leq \theta_{e, \max }, \theta_{e, \max }=4 \pi$ and $\theta_{e 2}=\theta_{e 1}-\pi / 2$. The second equiangular arm is defined by the same expressions except for the angle $\theta_{e 1}$, which now sweeps the angles from $\pi$ to $\theta_{e, \max }+\pi$. Similarly, the edges of the arms of the printed Archimedean spiral are described by

$$
r_{a 1}=\alpha_{a}\left(\theta_{a}-\theta_{e, \max }\right) r_{0} \exp \left(\alpha_{e} \theta_{e, \max }\right)
$$




$$
r_{a 2}=\alpha_{a}\left(\theta_{a}-\theta_{e, \max }\right)\left[r_{0} \exp \left(\alpha_{e} \theta_{e, \max }\right)-w\right]
$$

where $\alpha_{a}=0.25, w=1 \mathrm{~mm}$ and $\theta_{e, \max } \leq \theta_{a} \leq 20 \pi$. The shape parameters of the equiangular and Archimedean spiral portions were optimized simultaneously for best impedance match from $500 \mathrm{MHz}$ to $5 \mathrm{GHz}$. The optimization was first applied to the spiral structure without the cavity and then re-optimized in the presence of the cavity. As shown in Section 3, an impedance match better than $10 \mathrm{~dB}$ reflection loss was actually achieved from $350 \mathrm{MHz}$ to $5.5 \mathrm{GHz}$. The Archimedean portion of the spiral is critical in achieving this wideband impedance match while keeping the antenna diameter below $360 \mathrm{~mm}$. This is because the Archimedean shape offers significant length extension of the spiral arms within the available space, ensuring gradual current decay toward the arms' ends. This gradual decay is particularly important in the presence of the cavity, the walls of which are very close to the ends of the spiral arms. It has minimal impact on the antenna efficiency when an FR-4 substrate is used. As shown in Section 3, the loss contribution of the FR-4 material is far greater than the loss contribution of the copper traces forming the spiral (including the Archimedean portion). Likewise, the gain of the antenna is fairly insensitive to the proportion of the area occupied by the Archimedean spiral within the overall available area as long as this proportion ensures the required impedance match.

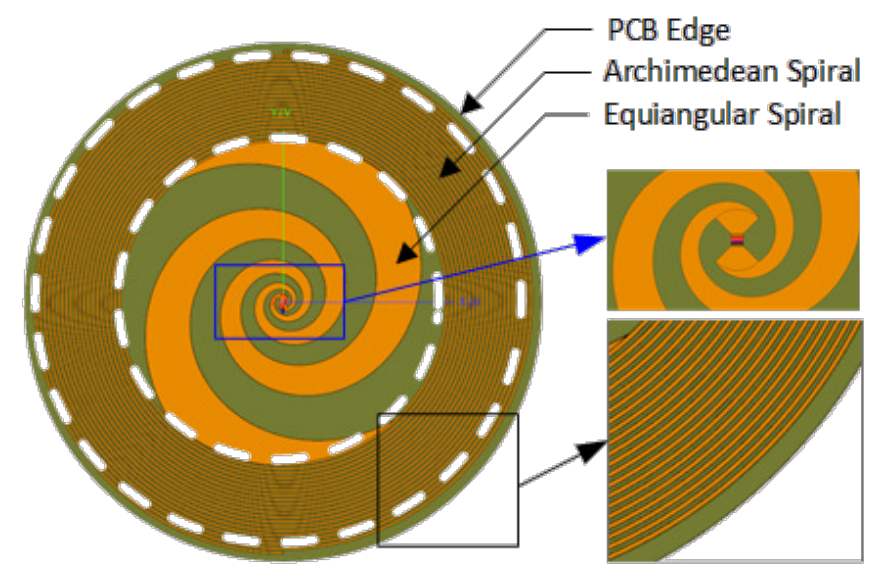

Figure 2. Planar view of the compound-spiral antenna structure. Enlarged views show in greater detail the balanced antenna port (top inset) and the fine structure of the outer Archimedean spiral (bottom inset).

\subsection{Center Raised Cylindrical Cavity}

In order to suppress the backward radiation, the compound spiral is backed by a metallic cavity. As discussed in Section 1, cavities negatively impact the antenna's broadband impedance, AR and gain patterns $[18,19,27,28]$. Mitigation strategies include absorbing materials inside the cavity or termination resistors $[21,35,36]$, which reduce the antenna efficiency. Here, we propose an absorber-free center-raised cylindrical cavity (see Figure 1) which is easy to fabricate due to its simple geometry. It significantly improves the front-to-back ratio. The compound spiral was optimized together with the cavity in order to preserve the wideband impedance match. We should mention that, without the cavity, the spiral achieves even wider impedance bandwidth from $300 \mathrm{MHz}$ all they way up to $6 \mathrm{GHz}$. However, with the cavity, the impedance match is compromised even within the specified bandwidth from $500 \mathrm{MHz}$ to $5 \mathrm{GHz}$, which necessitates an additional optimization cycle where the spiral and the cavity shape parameters are tuned simultaneously. The underlying design principle employs a reflecting surface at a distance of about $\lambda_{\text {min }} / 4$ ( $\lambda$ is wavelength) from the antenna center, where the higher frequencies are radiated, whereas the reflector is at about $\lambda_{\text {max }} / 4$ from the spiral elsewhere. This design principle can further be utilized to improve performance across the required bandwidth by having multiple raised sections, each corresponding to a $\lambda / 4$ distance from the spiral that relates to the radiated frequency at that section. This does however increase complexity and hence fabrication 
costs. An additional advantage of the center-raised cylinder is that it houses the feed balun on the outside (see Figure 1) thus shielding it from the antenna.

The dimensions for the center-raised cylindrical cavity (refer to Figure 1) are: $h_{1}=126 \mathrm{~mm}$ (cavity height), $h_{2}=105 \mathrm{~mm}$ (height of raised cylinder), $D_{1}=364 \mathrm{~mm}$ (cavity diameter including the rim) and $D_{2}=106 \mathrm{~mm}$ (diameter of the center-raised cylinder). It should be noted that neither the cavity height nor the raised cylinder height correspond to the exact theoretical $\lambda / 4$ values that relate to the minimum and maximum frequencies, but were rather optimized to obtain the best performance across the full frequency band.

\subsection{Balun}

A printed balun is designed to transform an unbalanced (coaxial) $50 \Omega$ feed to the spiral's balanced port, the simulated impedance of which varies from about $120-j 10 \Omega$ at low frequencies to about $150+j 20 \Omega$ at high frequencies. It consists of five linear-taper metallic sections at the bottom and the top of a $1.6 \mathrm{~mm}$ thick FR-4 PCB (see Figure 3). The balun design relies on a gradual transition from the balanced to unbalanced port to obtain an acceptable wide-band impedance match where a more gradual transition translates to a higher bandwidth. This gradual transition was found to be most effectively obtained within the physical length constraints through the use of differently tapered sections. Due to the varying input to output impedance ratios from the lower to higher frequencies, the structure needs to be tuned through simulation within the whole antenna, spiral and cavity included, to obtain an optimal impedance match. Section 1 is a short $50 \Omega$ microstrip line interfacing the coaxial port. Its bottom (ground) layer's width is five times the strip width. The balanced port of the antenna interfaces section 7, which is a short symmetrical strip line [40]. Sections 2-6 consist of top and bottom linearly tapered traces which provide the impedance transformation. Table 1 lists the shape parameters of the balun's sections. The design is constrained to keep the balun's total length equal to $h_{1}=126 \mathrm{~mm}$ so that it fits within the raised portion of the cavity.

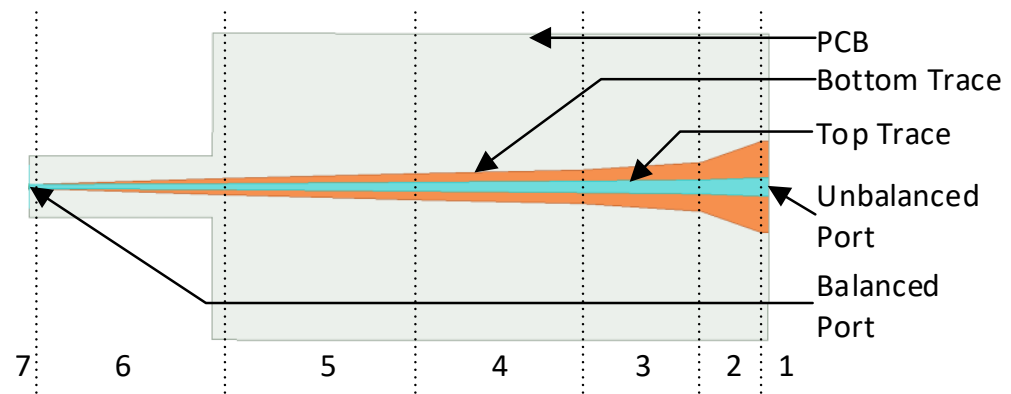

Figure 3. Planar view of the printed balun, consisting of seven sections (numbered at the bottom); for geometrical details see Table 1.

Table 1. Balun's shape parameters.

\begin{tabular}{cccc}
\hline Section & Length $(\mathbf{m m})$ & Gradient Top & Gradient Bottom \\
\hline 1 & 1.000 & 0.0000 & 0.0000 \\
2 & 10.00 & -0.0350 & -0.3500 \\
3 & 19.50 & -0.0103 & -0.0641 \\
4 & 29.25 & -0.0077 & -0.0222 \\
5 & 29.25 & -0.0077 & -0.0256 \\
6 & 31.00 & -0.1500 & -0.0323 \\
7 & 1.000 & 0.0000 & 0.0000 \\
\hline
\end{tabular}




\section{Results}

\subsection{Frequency-Domain Performance}

Figure 4 shows the fabricated antenna, which costs roughly 113 USD -23 USD for both the spiral antenna and balun and 90 USD for the cavity, to fabricate a unit. Figure 5 shows the measured and simulated (HFSS [38] and CST [39]) reflection coefficient versus frequency. It is better than $-10 \mathrm{~dB}$ from $350 \mathrm{MHz}$ to beyond $5 \mathrm{GHz}$. The three curves agree well at lower frequencies, but as the frequency increases, so too does the disagreement. This is attributed to the high uncertainty in the properties of the FR-4 substrate, which are not provided by the manufacturer in the GHz range. CST, which uses a transient solver, uses FR-4 parameters of $\epsilon_{r}=4.3$ and $\tan \delta=0.025$ at $10 \mathrm{GHz}$ in its library whereas HFSS, which uses a frequency domain solver, uses $\epsilon_{r}=4.4$ and $\tan \delta=0.02$ at $9.4 \mathrm{GHz}$.

The simulated input impedance at the balun's input ranges from $65-j 10 \Omega$ at low frequencies to $50+j 0 \Omega$ at high frequencies as compared to the respective values of $120-j 10 \Omega$ and $150+j 20 \Omega$ at the spiral's input.

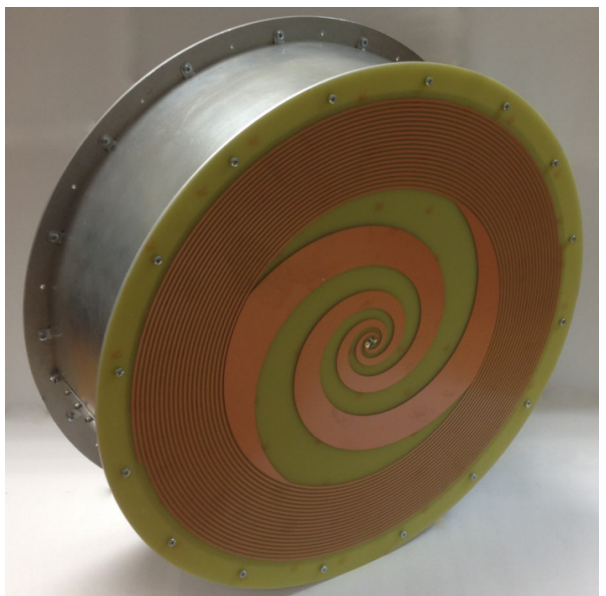

(a)

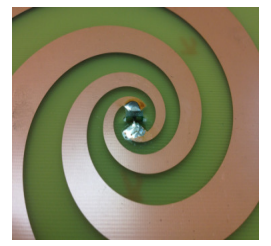

(b)

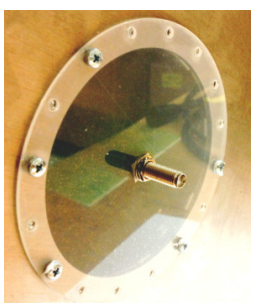

(c)

Figure 4. Assembled cavity-backed compound-spiral antenna: (a) front view; (b) spiral's feed; (c) raised portion of the cavity with the mounted balun.

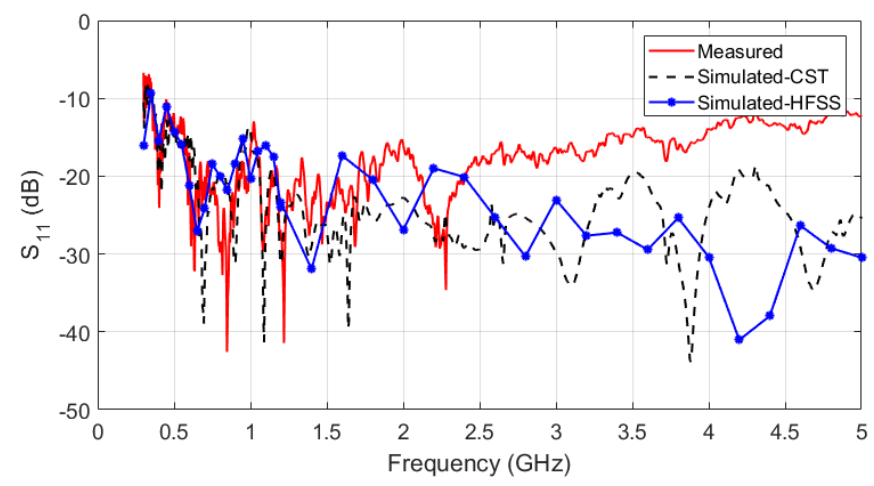

Figure 5. Measured and simulated reflection coefficient of the proposed antenna.

Far-field measurements were done in a commercial antenna-measurement facility. The measured total gain patterns are reported here in two elevation planes, $x z$ and $y z$ ( $z$ is along boresight). The elevation angle $\theta$ spans the range $[-\pi, \pi]$. Figure 6 shows the gain patterns at $0.5,2$ and $5 \mathrm{GHz}$. Pattern ripples close to boresight are observed above $1 \mathrm{GHz}$. Simulations confirm the effect to be due to the cavity, i.e., no ripples are observed if the cavity is removed. Pattern distortions due to loss-free reflectors are well-known [27] and the ripple effect is expected. Finally, Figure 7 shows 
a comparison of simulated and measured gain patterns at sample frequencies. Good agreement is observed especially within the cone defined by an elevation angle of 45 degrees.

This antenna is developed for a concealed-weapon detection close-range radar, where gain is important not only at boresight but within $\pm 45^{\circ}$ from it, translating into illuminating a person of about $1.8 \mathrm{~m}$ height at about $1 \mathrm{~m}$ stand-off distance. Within this beamwidth, the maximum total gain varies from about $5 \mathrm{dBi}$ to about $8 \mathrm{dBi}$ in the whole frequency band (see Table 2). The boresight total gain values are also shown in Table 2 . The maximum gain values are significantly higher than those reported in the literature for cavity-backed spirals with impedance bandwidths close to 10:1, e.g., 3.4 to $6.1 \mathrm{dBi}$ in [28] (simulated), -10 to $6 \mathrm{dBi}$ in [26], -2.5 to $5 \mathrm{dBi}$ in [24] and -1.77 to $2.37 \mathrm{dBi}$ in [23]. We note, however, that these designs employ absorbing or EBG cavities, mitigating the ripples in the gain patterns and implying that the maximum reported gain is that at boresight. The boresight gain in Table 2 is similar or only slightly better in comparison.

Table 2. Maximum and boresight measured total gain.

\begin{tabular}{ccccccc}
\hline Frequency $(\mathrm{GHz})$ & 0.5 & 1.0 & 2.0 & 3.0 & 4.0 & 5.0 \\
\hline Maximum $(\mathrm{dBi})$ & 5.8 & 4.71 & 8.55 & 8.27 & 6.71 & 7.86 \\
\hline Boresight $(\mathrm{dBi})$ & 5.8 & -2.75 & 2.07 & -0.14 & 0.0093 & 1.14 \\
\hline
\end{tabular}
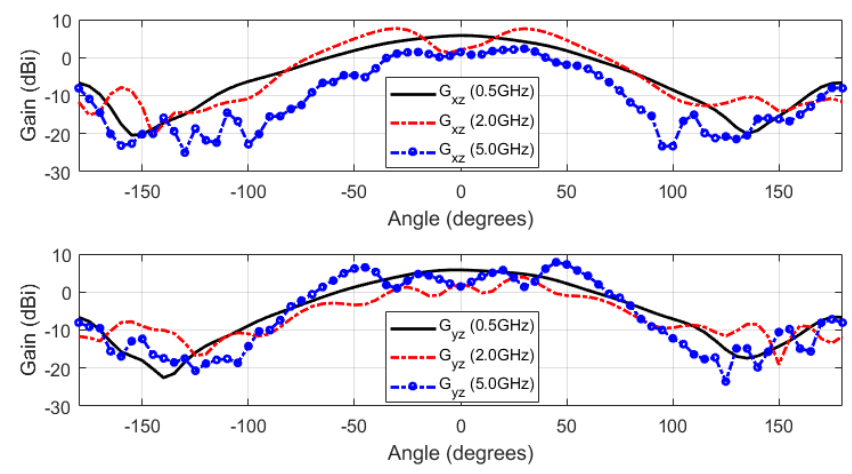

Figure 6. Measured total gain at $0.5,2$ and $5 \mathrm{GHz}$, plotted in two principal elevation planes, $x z$ and $y z$, where $z$ is along the antenna's boresight.

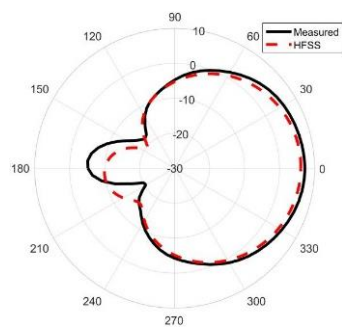

(a)

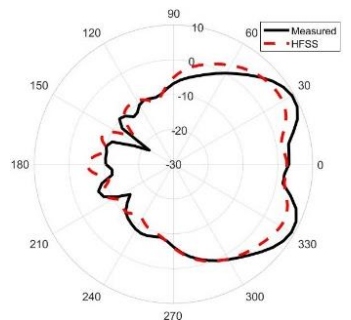

(b)

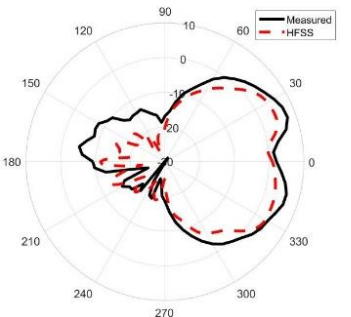

(c)

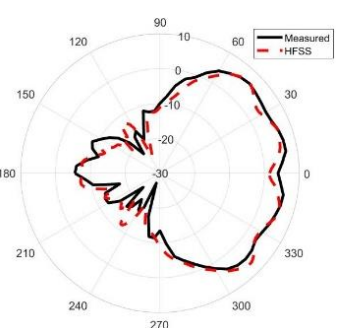

(d)

Figure 7. Sample plots comparing simulated and measured total gain patterns at: (a) 0.5 , (b) 2, (c) 4 and (d) $5 \mathrm{GHz}$. Patterns are plotted in the yz plane.

Figure 8 shows the front-to-back ratio (F/B) versus frequency. It is better than $8 \mathrm{~dB}$ from $0.5 \mathrm{GHz}$ to beyond $5 \mathrm{GHz}$. The F/B is calculated from the measured 3-D field patterns as the ratio of the total power in the forward half-space $\left(0^{\circ} \leq|\theta| \leq 90^{\circ}\right)$ and that radiated backwards $\left(90^{\circ}<|\theta| \leq 180^{\circ}\right)$.

Figure 8 also shows the measured antenna efficiency $e_{A}$, which is about $75 \%$ up to $2 \mathrm{GHz}$ after which it decreases steadily to $57 \%$ at $5 \mathrm{GHz}$. The efficiency is also obtained from simulations to compare the loss contribution of the FR-4 substrate to the conductor loss (copper with $\sigma=5.7 \cdot 10^{7} \mathrm{~S} / \mathrm{m}$ ). The substrate permittivity is set to 4.5 whereas $\tan \delta$ is set to $0.05,0.02$ and 0.0027 in three separate simulations. Here, $\tan \delta=0.0027$ corresponds to a low-loss substrate, e.g., Rogers $4003 \mathrm{C}$. It is evident 
that the losses are due mostly to the FR- 4 substrate, with $\tan \delta=0.02$ providing the closest match with measurements. It is also evident that utilizing this absorber-free cavity yields improved efficiency of $57 \%$ to $75 \%$ using a high loss FR-4 substrate as compared to $40 \%$ to $45 \%$ when using an absorptive backing and low loss substrates [1]. Simulation results further show that if a comparable low loss substrate is used in the proposed cavity, efficiency above $90 \%$ is achievable.

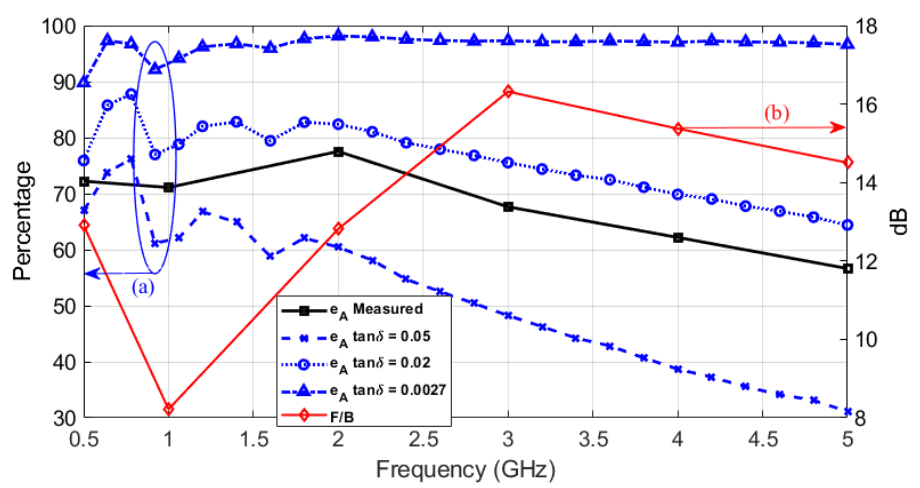

Figure 8. (a) Measured and simulated antenna efficiency $e_{A}$ versus frequency (in percentage) where the loss tangent of the substrate in simulations is set to: 0.05, 0.02 and 0.0027. (b) Measured front-to-back ratio $(\mathrm{F} / \mathrm{B})$ in $\mathrm{dB}$ versus frequency.

Figure 9 summarizes the results for the measured AR. Figure 9a shows the boresight and the minimum AR within $45^{\circ}$ of boresight versus frequency. The minimum AR is the highest $(4.6 \mathrm{~dB})$ at $0.5 \mathrm{GHz}$ and it is well below $1 \mathrm{~dB}$ at and beyond $1 \mathrm{GHz}$. Such AR values are comparable to the boresight AR of the spiral antennas employing absorbing or EBG cavities. However, similarly to the gain, the AR distribution varies within $45^{\circ}$ of boresight. In particular, the boresight $\mathrm{AR}$ is below $6 \mathrm{~dB}$ at all measured frequencies except at $4 \mathrm{GHz}$ where it is $7.5 \mathrm{~dB}$. To appreciate the range of variation, Figure $9 \mathrm{~b}-\mathrm{d}$ show the AR contour plots within $45^{\circ}$ from boresight at $0.5 \mathrm{GHz}, 4 \mathrm{GHz}$, and $5 \mathrm{GHz}$. The $4 \mathrm{GHz}$ plot is chosen in addition to the lowest and highest frequencies because it corresponds to the worst boresight AR. It is clear that the minimum AR value of $0.2 \mathrm{~dB}$ is close to boresight (within $6^{\circ}$ ) but the AR variation is anywhere between $0 \mathrm{~dB}$ and $9 \mathrm{~dB}$. As mentioned in the Introduction, absorber-free metallic cavities are known to distort the AR and gain patterns of spiral antennas [27]. The distortions appear in the form of ripples close to boresight, which ripples may shift the minimum AR (and maximum gain) off boresight.
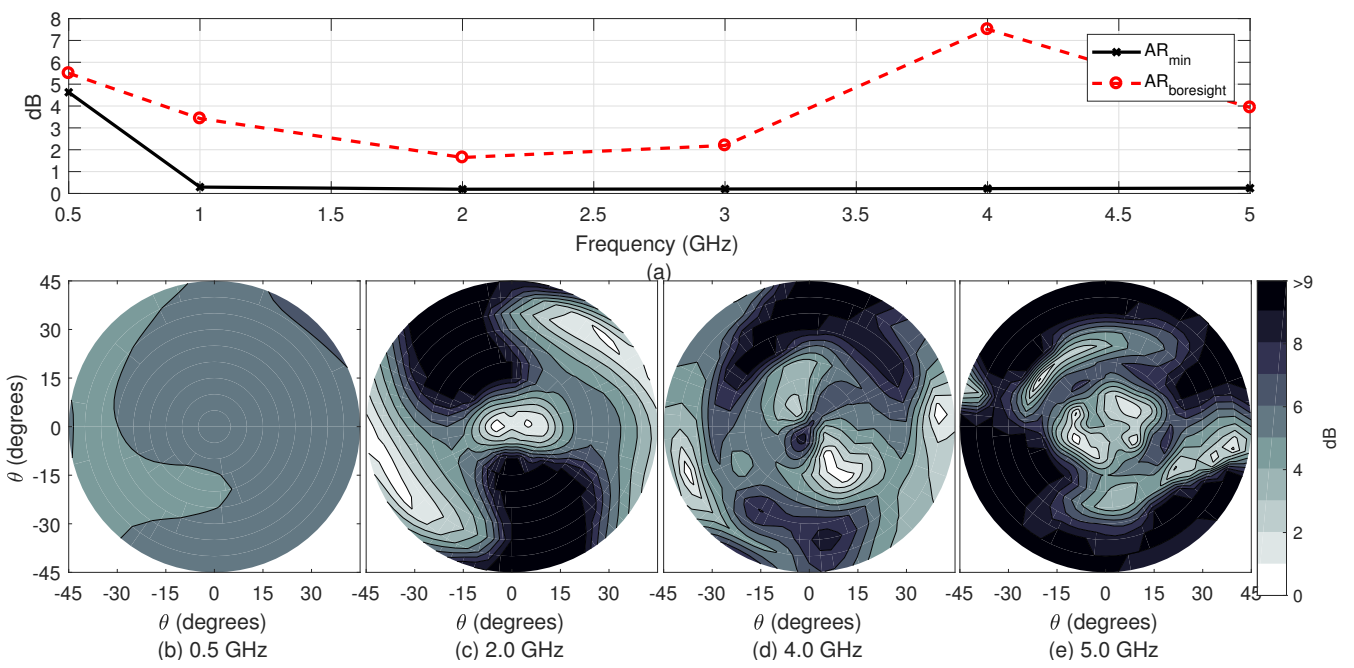

Figure 9. (a) Measured axial ratio (AR) at boresight and minimum (within $45^{\circ}$ of boresight) AR versus frequency. Contour plots of the measured AR at: (b) 0.5, (c) 2.0, (d) 4.0 and (e) $5.0 \mathrm{GHz}$. 
It is worth noting that the gain and AR distributions in the cone of $45^{\circ}$ off boresight show strong correlation between the directions of minimum gain and maximum AR.

To illustrate further the polarization performance of the antenna, Figure 10 shows plots of the right-hand circular polarization (RHCP) and left-hand circular polarization (LHCP) gain patterns at sample frequencies in the $x z$ plane.
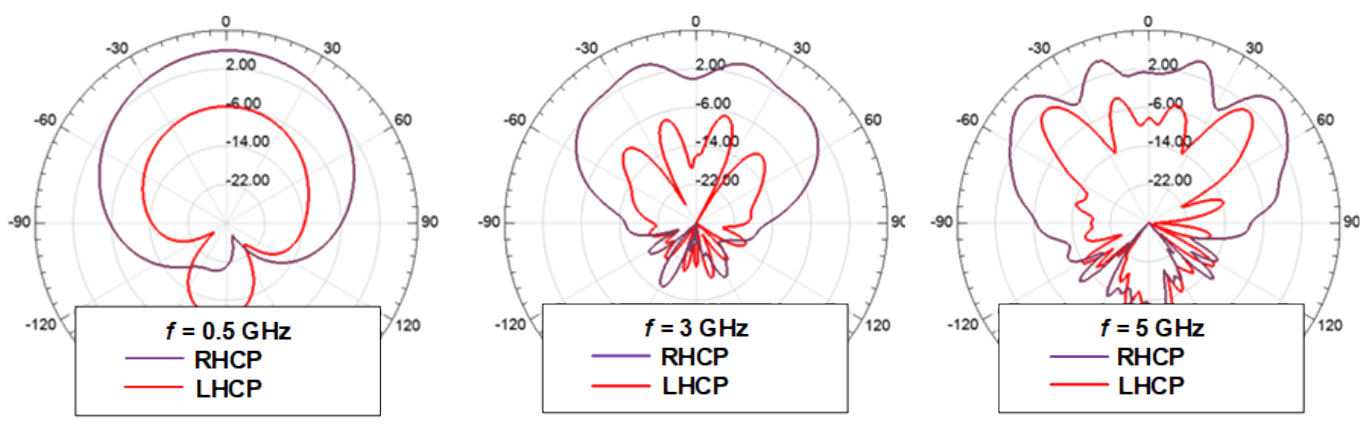

Figure 10. Sample plots comparing the right-hand circular polarization (RHCP) and left-hand circular polarization (LHCP) gain patterns in the $x z$ plane at: $0.5,3$ and $5 \mathrm{GHz}$.

\subsection{Time-Domain Performance}

The fidelity factor $F$ quantifies the dispersion characteristics of an antenna, i.e., the distortion it causes on a broadband signal upon transmission or reception [41]. It is calculated as the maximum cross-correlation between the transmitted $v_{t}(t)$ and received $v_{r}(t)$ time-domain signals [42]:

$$
F=\max \left[\frac{\int_{-\infty}^{+\infty} v_{t}(t) \cdot v_{r}(t-\tau) d t}{\sqrt{\int_{-\infty}^{+\infty}\left|v_{t}(t)\right|^{2} d t \cdot \int_{-\infty}^{+\infty}\left|v_{r}(t)\right|^{2} d t}}\right]
$$

To obtain $F$ through measurements, the approach in [42] was used. It employs two identical antennas aligned along boresight. One antenna transmits with the excitation signal $v_{e}(t)$ at its input. The signal received at the terminals of the second antenna is $v_{r}(t)$. The excitation $v_{e}(t)$ is measured directly at the output of the signal generator. The transmitted signal $v_{t}(t)$ is extracted in the frequency domain using [42]:

$$
V_{t}(\omega)=\sqrt{j 2 k_{0} d \cdot V_{e}(\omega) \cdot V_{r}(\omega) e^{j k_{0} d}}
$$

where $V_{t}(\omega), V_{r}(\omega)$ and $V_{e}(\omega)$ are the Fourier transforms of $v_{t}(t), v_{r}(t)$ and $v_{e}(t)$, respectively, $k_{0}$ is the free-space wavenumber and $d$ is the distance between the antennas.

In our measurement setup, two identical spiral antennas were positioned facing each other in an anechoic chamber with a separation $d=4.2 \mathrm{~m}$ (limited by the chamber size) as shown in Figure 11. A generator similar to the one in [43] provides excitation with a differentiated Gaussian pulse with a $10 \mathrm{~dB}$ bandwidth from $450 \mathrm{MHz}$ to $4.8 \mathrm{GHz}$. The received signal $v_{r}(t)$ and the excitation signal $v_{e}(t)$ were measured with a Keysight InfiniiVision 6000 X-Series oscilloscope. Figure 12 shows $v_{r}(t)$ and $v_{e}(t)$ along with the calculated $v_{t}(t)$ after normalization and alignment for maximum correlation. Using (5) and (6), the fidelity was calculated to be $F \approx 0.62$, which is comparable with that of other spiral antennas [42]. For reference, we note that the same setup was used to measure the fidelity of an UWB linearly polarized antipodal antenna with a similar bandwidth, with the result being $F \approx 0.85$. It is worth noting that no secondary pulse is observed in the received voltage $v_{r}(t)$ (see Figure 12) as the one reported in [16]. 


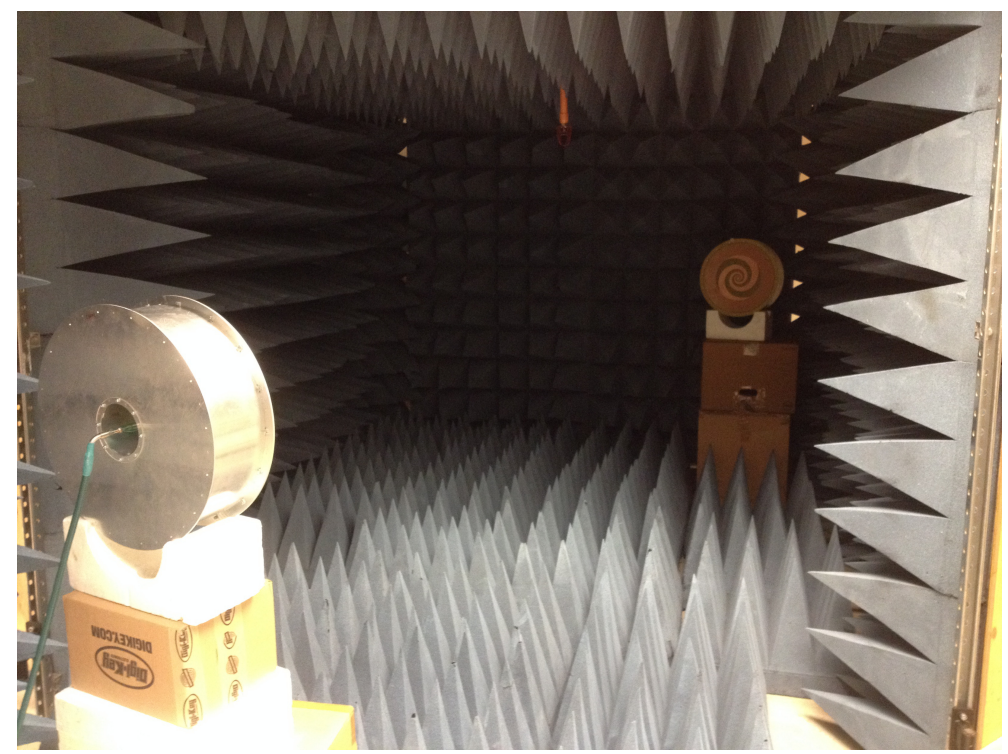

Figure 11. Antenna fidelity measurement setup.

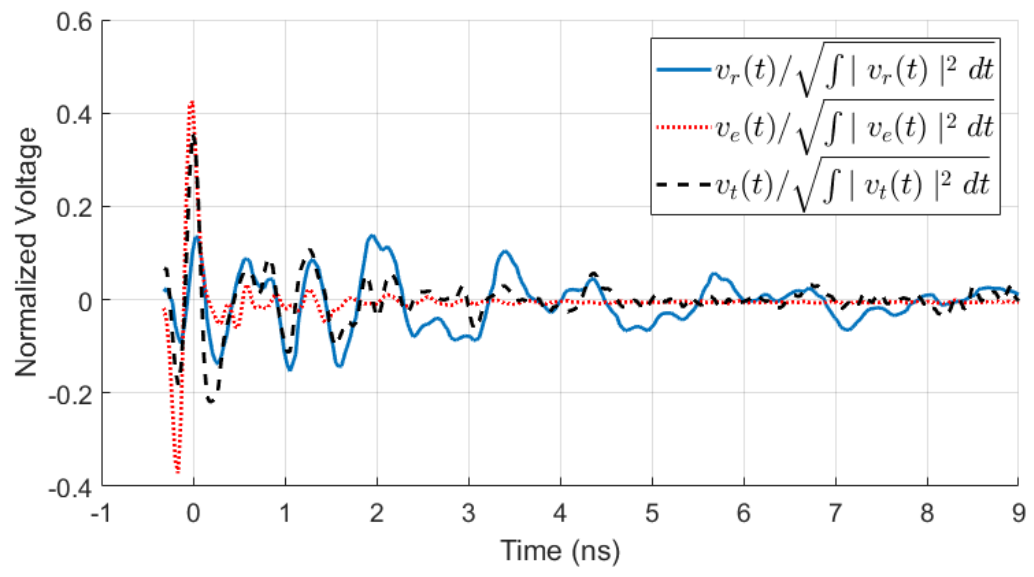

Figure 12. Normalized excitation, received and transmit signals after temporal alignment for maximum correlation.

\section{Conclusions}

A low-cost high-efficiency cavity-backed spiral antenna is proposed, which achieves: (i) 16:1 impedance bandwidth (from $350 \mathrm{MHz}$ to $5.5 \mathrm{GHz}$, at $10 \mathrm{~dB}$ reflection loss), (ii) front-to-back ratio better than $8 \mathrm{~dB}$, (iii) maximum gain within the cone of $45^{\circ}$ off boresight ranging from 5 to $8 \mathrm{dBi}$, and (iii) efficiency ranging from $72 \%$ at $500 \mathrm{MHz}$ to $57 \%$ at $5 \mathrm{GHz}$ despite the high loss of the FR-4 substrate. Simulations indicate that the efficiency is well above $90 \%$ if low-loss substrates are used (with ten-fold price increase). The maximum gain values are significantly higher than those reported in the literature for cavity-backed spirals with similar impedance bandwidths, e.g., $3.4 \mathrm{dBi}$ to $6.1 \mathrm{dBi}$ in [28] (simulated), $-10 \mathrm{dBi}$ to $6 \mathrm{dBi}$ in [26], $-2.5 \mathrm{dBi}$ to $5 \mathrm{dBi}$ in [24] and $-1.77 \mathrm{dBi}$ to $2.37 \mathrm{dBi}$ in [23]. Efficiency is also significantly higher than that typically reported for spiral antennas with absorptive backing, e.g., $40 \%$ to $45 \%$ [20,37]. These performance metrics are superior to reported cavity-backed spirals and they prove that absorber-free center-raised cavities can ensure unidirectional patterns while maintaining extremely wide impedance bandwidths. In terms of AR and fidelity, the proposed design is comparable to existing absorber-free reflector-backed spirals. The drawback of absorber-free cavities is in the AR and gain pattern distortions (ripples close to boresight), which may shift the directions of maximum gain and minimum AR off boresight. Such distortions are acceptable in the close-range application for which the antenna is designed (concealed-weapon detection radar system [44,45]). 
However, if patterns with a single maximum along boresight are required, the use of absorbers or terminating resistors is necessary. Such mitigation strategies are certainly applicable to the current design. It is also worth noting that the size of the antenna is comparable to that of reported UWB spirals. Its diameter as a fraction of the largest wavelength is 0.425 , which is comparable to: 0.404 in [23], 0.336 in [24], 0.434 in [26] and 0.432 in [28].

Author Contributions: Conceptualization, Y.L.; methodology, Y.L.; validation, C.B.; formal analysis, C.B.; investigation, C.B.; resources, Y.L.; writing—original draft preparation, C.B.; writing-review and editing, C.B.; supervision, N.N.; project administration, N.N.; funding acquisition, N.N. All authors have read and agreed to the published version of the manuscript.

Funding: This research was funded by Natural Sciences and Engineering Research Council of Canada (NSERC), grant CRDPJ 522367-17, the Ontario Centres of Excellence (OCE) grant OCE VIP II \#30493, and Patriot One Tech. Inc.

Conflicts of Interest: The authors declare no conflict of interest.

\section{References}

1. Sachs, J. Handbook of Ultra-Wideband Short-Range Sensing: Theory, Sensors, Applications; Wiley-VCH Verlag \& Co. KGaA: Weinheim, Germany, 2012.

2. Nguyen, C.; Han, J. Time-Domain Ultra-Wideband Radar, Sensor and Components; Springer: Berlin/Heidelberg, Germany, 2014.

3. Taylor, J.D. Ultrawideband Radar: Applications and Design; CRC Press: Boca Raton, FL, USA, 2016.

4. Dyson, J.D. A survey of the very wide band and frequency independent antennas-1945 to the present. J. Res. Nat. Bur. Stand.-(Radio Propag.) 1962, 66, 1-6. Available online: https://nvlpubs.nist.gov/nistpubs/ jres/66d/jresv66dn1p1_a1b.pdf (accessed on 27 August 2020). [CrossRef]

5. Curtis, W. Spiral antennas. IRE Trans. Antennas Propag. 1960, 8, 289-306. [CrossRef]

6. Rumsey, V.H. Frequency Independent Antennas; Academic Press: Cambridge, MA, USA, 1966. .

7. Corzine, R.G.; Mosko, J.A. Four-Arm Spiral Antennas; Artech House: Norwood, MA, USA, 1990.

8. DuHamel, R.H.; Chadwick, G.G. Frequency-independent antennas. In Antenna Engineering Handbook, 2nd ed.; Johnson, R.C., Jasik, H., Eds.; McGraw-Hill Book Company: New York, NY, USA, 1984; Chapter 14 , pp. 14-16-14-26.

9. Filipović, D.S.; Cencich, T. Frequency-independent antennas. In Antenna Engineering Handbook, 4th ed.; Volakis, J.L., Ed.; Mc-Graw-Hill: New York, NY, USA, 2007; Chapter 13.

10. Nakano, H. Frequency-independent antennas: Spirals and log-periodics. In Modern Antenna Handbook; Balanis, C.A., Ed.; Wiley Online Library: Hoboken, NJ, USA, 2007; Chapter 6.

11. Stutzman, W.L.; Thiele, G.A. Antenna Theory and Design, 3rd ed.; Wiley: Hoboken, NJ, USA, 2013.

12. Balanis, C.A. Antenna Theory: Analysis and Design, 4th ed.; Wiley: Hoboken, NJ, USA, 2013.

13. Sheen, D.; McMakin, D.; Hall, T. Near-field three-dimensional radar imaging techniques and applications. Appl. Opt. 2010, 49, E83-E93. [CrossRef] [PubMed]

14. Volakis, J.L.; Nurnberger, M.W.; Filipović, D.S. Broadband cavity-backed slot spiral antenna. IEEE Antennas Propag. Mag. 2001, 43, 15-26. [CrossRef]

15. McFadden, M.; Scot, W. Analysis of the equiangular spiral antenna on a dielectric substrate. IEEE Trans. Antennas Propag. 2007, 55, 3163-3171. [CrossRef]

16. McFadden, M. Analysis of the Equiangular Spiral Antenna. Ph.D. Thesis, School of Electrical and Computer Engineering, Georgia Institute of Technology, Atlanta, GA, USA, 2009.

17. Fumeaux, C.; Baumann, D.; Vahldieck, R. Finite-volume time-domain analysis of a cavity-backed Archimedean spiral antenna. IEEE Trans. Antennas Propag. 2006, 54, 844-851. [CrossRef]

18. Nakano, H.; Nogami, K.; Arai, S.; Mimaki, H.; Yamauchi, J. A spiral antenna backed by a conducting plane reflector. IEEE Trans. Antennas Propag. 1986, 34, 791-796. [CrossRef]

19. Nakano, H.; Soga, H.; Honma, T.; Yamauchi, J. Effects of adding a small disk to a spiral antenna backed by a conducting plane reflector. IEE Proc. H Microwaves Antennas Propag. 1991, 138, 375-377. [CrossRef]

20. Nakano, H.; Kikkawa, K.; Iitsuka, Y.; Yamauchi, J. Equiangular spiral antenna backed by a shallow cavity with absorbing strips. IEEE Trans. Antennas Propag. 2008, 56, 2742-2747. [CrossRef] 
21. Serhir, M.; Guinvarc'h, R. A low-profile cavity-backed dual-polarized spiral antenna array. IEEE Antennas Wirel. Propag. Lett. 2013, 12, 524-527. [CrossRef]

22. Sammeta, R.; Filipović, D.S. Quasi frequency-independent increased bandwidth planar log-periodic antenna. IEEE Trans. Antennas Propag. 2014, 62, 1937-1944. [CrossRef]

23. Sheikh, Z.; Tahir, F.A. Gain enhancement of low profile cavity backed spiral antenna at low frequencies. In Proceedings of the Progress of the 2017 Progress in Electromagnetics Research Symposium-Fall (PIERS-FALL), Singapore, 19-22 November 2017; pp. 1771-1773.

24. Hamza, M.; Khan, W.T. Hybrid utilization of loading techniques and cavity groove for performance enhancement of the UWB (2-18 GHz) spiral antenna. Int. J. Antennas Propag. 2018, 2018, 9167154. [CrossRef]

25. Acree, M.A.; Prata, A. Archimedean spiral-mode microstrip antenna with improved axial ratio. In Proceedings of the IEEE AP-S/URSI International Symposium on Antennas and Propagation, Orlando, FL, USA, 11-16 July 1999; Volume 2, pp. 1232-1235.

26. Liu, C.; Lu, Y.; Du, C.; Cui, J.; Shen, X. The broadband spiral antenna design based on hybrid backed-cavity. IEEE Trans. Antennas Propag. 2010, 58, 1876-1882.

27. Buck, M.C.; Filipović, D.S. Spiral cavity backing effects on pattern symmetry and modal contamination. IEEE Antennas Wirel. Propag. Lett. 2006, 5, 243-246. [CrossRef]

28. Ding, F.; Zhang, F.; Zhang, Y. The broadband composite structure spiral antenna with a ladder-shaped backed-cavity. In Proceedings of the 2011 4th IEEE International Symposium Microwave, Antenna, Propagation and EMC Technologies for Wireless Communications, Beijing, China, 1-3 November 2011; pp. 120-123.

29. Shire, A.M.; Jahun, K.I.; Ashwal, W.A.; Jenu, M.Z.M.; Ramli, K.N. Effects of cavity ground plane on UWB Archimedean spiral antenna. In Proceedings of the 2015 IEEE International Conference on Computer, Communication, and Control Technology (I4CT 2015), Sarawak, Malaysia, 21-23 April 2015.

30. Kim, H.-B.; Hwang, K.-C.; Kim, H-S. Cavity-backed two-arm spiral antenna with a ring-shaped absorber for partial discharge diagnosis. J. Electr. Eng. Technol. 2013, 8, 856-862. [CrossRef]

31. Louertani, K.; Chio, T.-H. Hybrid equi-angular to Archimedean spiral antenna. In Proceedings of the 2012 IEEE International Symposium on Antennas and Propagation, Chicago, IL, USA, 8-14 July 2012.

32. Wang, Y.; Wang, G.; Yu, Z. Design of a highly miniaturized compound spiral antenna. In Proceedings of the International Symposium on Signals, Systems and Electronics, Nanjing, China, 16-19 September 2010.

33. Zhao, Y.; Hu, W. Design of a UWB unidirectional radiation compound spiral antenna. In Proceedings of the IEEE 6th International Symposium on Microwave, Antenna, Propagation, and EMC Technologies (MAPE), Shanghai, China, 28-30 October 2015.

34. Chen, M.; Wang, G.; Zhou, S. Novel compound planar spiral antenna. In Unifying Electrical Engineering and Electronics Engineering: Proceedings of the 2012 International Conference on Electrical and Electronics Engineering; Springer: Berlin/Heidelberg, Germany, 2013; pp. 1493-1500.

35. Maldonado-Vargas, J.; Rodríguez-Solís, R.A.; Elmansouri, M.A.; Filipovic, D.S. A UWB cavity-backed compound power-Archimedean slot spiral for body centric wireless communications applications. In Proceedings of the IEEE AP-S/URSI International Symposium on Antennas and Propagation, Vancouver, BC, USA, 19-25 July 2015; pp. 1978-1979.

36. Zhu, Y.-X.; Zhong, S.-S.; Xu, S.-Q. Miniaturized compound spiral slot antenna. Microw. Opt. Tech. Lett. 2008, $50,2799-2801$.

37. Elmansouri, M.A.; Filipovic, D.S. Low-dispersion spiral antennas. IEEE Trans. Antennas Propag. 2012, 60, 5522-5530. [CrossRef]

38. ANSYS HFSS (19.2.0), ANSYS. Available online: https:/ / www.ansys.com/products/electronics/ansys-hfss (accessed on 17 September 2019).

39. CST Studio Suite. Dassault Systèmes. 6-15 June 2018. Available online: https:/ /www.3ds.com/productsservices/simulia/products / cst-studio-suite/ (accessed on 17 September 2019).

40. Wheeler, H.A. Transmission-line properties of parallel strips separated by a dielectric sheet. IEEE Trans. Microw. Theory Tech. 1965, 13, 172-185. [CrossRef]

41. Quintero, G.; Zurcher, J.; Skrivervik, A. System fidelity factor: A new method for comparing UWB antennas. IEEE Trans. Antennas Propag. 2011, 59, 2502-2512.

42. Elmansouri, M.; Radway, M.; Filipović, D. Frequency- and time-domain performance of four-arm mode-2 spiral antennas. IEEE Trans. Antennas Propag. 2012, 60, 2627-2634. [CrossRef] 
43. Pitcher, A.D.; McCombe, J.J.; Eveleigh, E.A.; Nikolova, N.K. Compact transmitter for pulsed-radar detection of on-body concealed weapons. In Proceedings of the IEEE MTT-S International Microwave Symposium, Philadelphia, PA, USA, 10-15 June 2018.

44. Nikolova, N.K.; Thayaparan, T. Ultra-Wideband (UWB) High-Resolution Noise Radar for Concealed Weapon Detection. Tech. Report DRDC-Ottawa TR 2013-160, Defence Research and Development Canada. February 2014. Available online: https://cradpdf.drdc-rddc.gc.ca/PDFS/unc231/p804019_A1b.pdf (accessed on 27 August 2020).

45. Nikolova, N.K.; McCombe, J.J. On-Body Concealed Weapon Detection System. U.S. Patent No. 10,229,328, 12 March 2019. Available online: https:/ / patentimages.storage.googleapis.com/0f/d5/1e/a1a32e2f905ec5/ US20150379356A1.pdf (accessed on 27 August 2020).

(C) 2020 by the authors. Licensee MDPI, Basel, Switzerland. This article is an open access article distributed under the terms and conditions of the Creative Commons Attribution (CC BY) license (http:// creativecommons.org/licenses/by/4.0/). 